University of Nebraska - Lincoln

DigitalCommons@University of Nebraska - Lincoln

Stephen Ducharme Publications

Research Papers in Physics and Astronomy

2-26-1998

\title{
Two-dimensional ferroelectric films
}

Alexander V. Bune

University of Nebraska at Lincoln

Vladimir M. Fridkin

Institute of Crystallography, Russian Academy of Sciences, Moscow, fridkin@ns.crys.ras.ru

Stephen Ducharme

University of Nebraska at Lincoln, sducharme1@unl.edu

Lev M. Blinov

Institute of Crystallography, Russian Academy of Sciences, Moscow

Serguei P. Palto

Institute of Crystallography, Russian Academy of Sciences, Moscow

See next page for additional authors

Follow this and additional works at: https://digitalcommons.unl.edu/physicsducharme

Part of the Condensed Matter Physics Commons, and the Materials Science and Engineering

Commons

Bune, Alexander V.; Fridkin, Vladimir M.; Ducharme, Stephen; Blinov, Lev M.; Palto, Serguei P.; Sorokin, Alexander V.; Yudin, S. G.; and Zlatkin, A., "Two-dimensional ferroelectric films" (1998). Stephen Ducharme Publications. 45.

https://digitalcommons.unl.edu/physicsducharme/45

This Article is brought to you for free and open access by the Research Papers in Physics and Astronomy at DigitalCommons@University of Nebraska - Lincoln. It has been accepted for inclusion in Stephen Ducharme Publications by an authorized administrator of DigitalCommons@University of Nebraska - Lincoln. 


\section{Authors}

Alexander V. Bune, Vladimir M. Fridkin, Stephen Ducharme, Lev M. Blinov, Serguei P. Palto, Alexander V. Sorokin, S. G. Yudin, and A. Zlatkin 


\title{
Two-dimensional ferroelectric films
}

\author{
A.V. Bune, ${ }^{1,2}$ V. M. Fridkin, ${ }^{1,2}$ Stephen Ducharme,' L. M. Blinov, ${ }^{2}$ \\ S. P. Palto, ${ }^{2}$ A.V. Sorokin, ${ }^{2}$ S. G. Yudin, ${ }^{2}$ and A. Zlatkin ${ }^{2}$ \\ ' Department of Physics and Astronomy and Center for Materials Research and Analysis, University of Nebraska-Lincoln, \\ Lincoln, Nebraska 68588-0I II, USA \\ ${ }^{2}$ Institute of Crystallography, Russian Academy of Sciences, I 17333 Moscow, Russia \\ Corresponding author — Stephen Ducharme, email sducharmel@unl.edu
}

\begin{abstract}
Ultrathin crystalline films offer the possibility of exploring phase transitions in the crossover region between two and three dimensions. Second-order ferromagnetic phase transitions have been observed in monolayer magnetic films [1,2], where surface anisotropy energy stabilizes the two-dimensional ferromagnetic state at finite temperature [3]. Similarly, a number of magnetic materials have magnetic surface layers that show a second-order ferromagnetic-paramagnetic phase transition with an increased Curie temperature [4]. Ferroelectricity is in many ways analogous to ferromagnetism, and bulk-like ferroelectricity and finite-size modifications of it have been seen in nanocrystals as small as $250 \AA$ in diameter [5], in perovskite films $100 \AA$ thick [6] and in crystalline ferroelectric polymers as thin as $25 \AA$ [7-10]. But these results can be interpreted as bulk ferroelectricity suppressed by surface depolarization energies, and imply that the bulk transition has a minimum critical size [ I I-13]. Here we report measurements of the ferroelectric transition in crystalline films of a random copolymer of vinylidene fluoride and trifluoroethylene just $10 \AA$ (two monolayers) thick. We see a first-order ferroelectric phase transition with a transition temperature nearly equal to the bulk value, even in these almost two-dimensional films. In addition, we see a second first-order transition at a lower temperature, which seems to be associated with the surface layers only. The near-absence of finite-size effects on the bulk transition implies that these films must be considered as two-dimensional ferroelectrics.
\end{abstract}

Ferroelectric polymers have been studied in great detail for nearly 30 years and are in wide use in a variety of piezoelectric devices $[14,15]$. One of the most interesting systems is the random copolymer of vinylidene fluoride with trifluoroethylene, $\mathrm{P}(\mathrm{VDF}-\mathrm{TrFE})[16]$, consisting of $-\left(\left(-\mathrm{CF}_{2}-\mathrm{CH}_{2}\right)_{x}-\left(-\mathrm{CF}_{2}-\right.\right.$ (CHF- $\left.)_{1-x}\right) n$ - chains with a regular intra-chain period of $2.6 \AA$, controlled by the $\mathrm{C}-\mathrm{C}$ bonds between fluorine pairs, as shown in Figure 1a. In the ferroelectric phase, the all-trans chains are arranged in parallel rows in a quasi-hexagonal close packing with orthorhombic mm2 structure [17]. P(VDF-TrFE 70:30) has a first-order ferroelectric-paraelectric phase transition (connected with the conversion of all-trans chains to mixtures of trans and gauche bonds with little or no net dipole moment [16] with Curie temperature $T_{c} \approx 100{ }^{\circ} \mathrm{C}$ (the Curie temperature increases with the proportion of the VDF component), thermal hysteresis of $\Delta T_{c} \approx 20{ }^{\circ} \mathrm{C}$, and a spontaneous polarization $P_{\mathrm{s}}$ $\approx 0.1 \mathrm{C} \mathrm{m}^{-2}$ at room temperature [16]. Thin films have usually been formed by solvent spinning and are polymorphous, containing amorphous material and incompletely orientated crystallites. Finite-size effects were evident from an increase in the coercive field in films as thin as $600 \AA$ [18].

The first crystalline Langmuir-Blodgett-deposited polymer films, of $\mathrm{P}(\mathrm{VDF}-\mathrm{TrFE} 70: 30)$, achieved a great improvement in quality $[7,8,10]$. They have excellent crystalline order and show the first-order ferroelectric-paraelectric transition at $T_{\mathrm{c}} \approx 80{ }^{\circ} \mathrm{C}$ on cooling. These ferroelectric polymer films, 30 monolayers (ML) thick ( 150 ̊), also showed double hys- teresis and the critical point [19], and a new conductance switching controlled by the polarization state [8]. Finite-size effects have been demonstrated in these films; the coercive field scaled as the -0.7 power of the thickness in films with 35-135 ML [9].

For the present work we first recorded an atomic-resolution image of a single-monolayer P(VDF-TrFE 70:30) film on graphite by scanning tunnelling microscopy (STM, Figure 1b) showing the excellent short-range order and uniform orientation of all the all-trans polymer chains. Figure $2 \mathrm{a}-\mathrm{c}$ shows that multilayer ferroelectric Langmuir-Blodgett-deposited polymer (FLP) films with aluminium electrodes have the dielectric anomalies and thermal hysteresis that mark the usual bulk first-order ferroelectric phase transition found at $77^{\circ} \mathrm{C}$ on cooling $\left(108{ }^{\circ} \mathrm{C}\right.$ on heating) in the $30-\mathrm{ML}$ film and decreasing to $68^{\circ} \mathrm{C}\left(98^{\circ} \mathrm{C}\right)$ in the 2-ML film. The pyroelectric response shown in Figure 3 also records the bulk transition near $73^{\circ} \mathrm{C}$, though the precise transition temperature is not as clear as in the dielectric measurements. Therefore, the "bulk" first-order phase transition at $\sim 80^{\circ} \mathrm{C}$ remains even in the 2-ML films (Figure $2 \mathrm{c}$ ) $-\mathrm{a}$ result that challenges theoretical predictions that the transition temperature should decrease, and ferroelectricity vanish, in films below a minimum critical thickness [11-13]. FLP films of $30 \mathrm{ML}$ or less all have approximately the same room-temperature relative dielectric constant $\varepsilon=8$ \pm 2 , as shown in Figure $2 \mathrm{~d}$, approximately one-half the value found in thick spun films. 
The hysteresis loops shown in Figure 4 show good saturation for the 5-ML and 30-ML films and incomplete saturation for the 2-ML film. The 5-ML and 2-ML films show considerable bias, probably due to interactions with the substrate or the top electrode, consistent with our earlier observations on the dynamics of switching FLP films [8,9]. The coercive fields of $0.9 \pm 0.2 \mathrm{GV} \mathrm{m}^{-1}$ (30 ML), $0.6 \pm 0.1 \mathrm{GV} \mathrm{m}^{-1}(5 \mathrm{ML})$, and $>0.5$ $\mathrm{GV} \mathrm{m}^{-1}(2 \mathrm{ML})$ are far larger than in other ferroelectric materials and are closely approaching the intrinsic coercive field $P_{\mathrm{s}} / \varepsilon \varepsilon_{0} \approx 1 \mathrm{GV} \mathrm{m}{ }^{-1}$ expected in the absence of nucleation, consistent with the very high crystallinity (here $\varepsilon_{0}$ is the permittivity of free space). The films achieved at least $50 \%$ of the maximum polarization expected if all dipoles are aligned perpendicular to the film, but our data could not rule out a tendency towards in-plane polarization as is observed in ultrathin magnetic films [20].

Figures 2 and 3 also show a new and unexpected phase transition at a lower temperature of $\sim 20^{\circ} \mathrm{C}$ in films of $30 \mathrm{ML}$ or less. The dielectric constant (Figure 2) shows peaks at $\sim 20$ ${ }^{\circ} \mathrm{C}$ on cooling $\left(28^{\circ} \mathrm{C}\right.$ on heating) in the pyroelectric response (Figure 3) in the 5-ML and 2-ML films. The polarization hysteresis loops from films of 30,5, and 2 ML (Figure 4) show that ferroelectric switching is obtained at $25^{\circ} \mathrm{C}$ in all the FLP films, even those as thin as $2 \mathrm{ML}$. We attribute the behavior at $\sim 20^{\circ} \mathrm{C}$ to a new first-order ferroelectric transition in the surface lay-
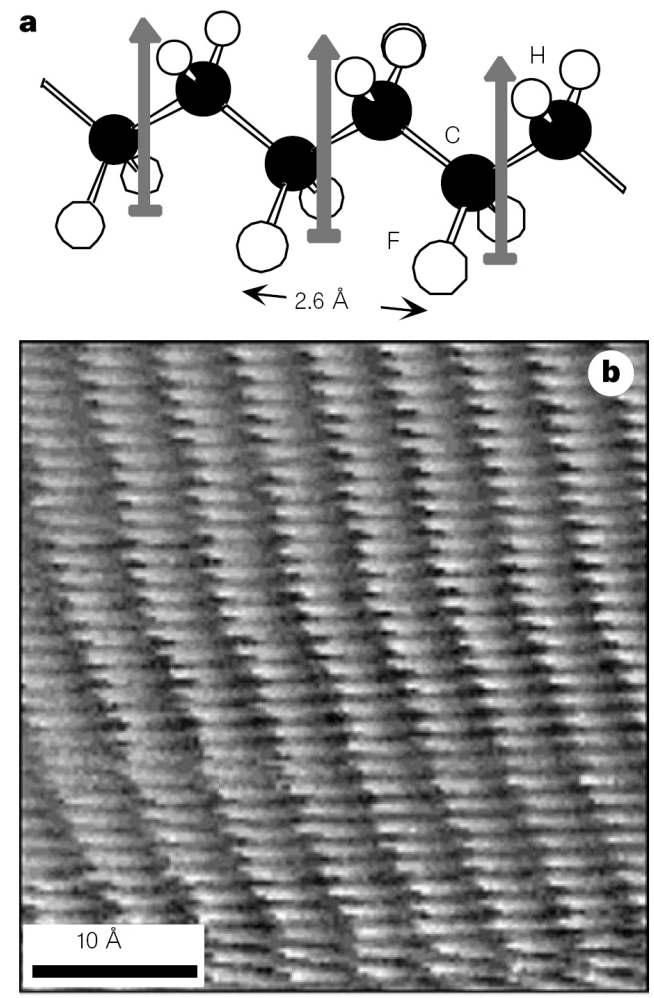

Figure I. Structure of the polymer chains and films. a) Fragment of a P(VDFTrFE 70:30) copolymer chain. $30 \%$ of the VDF $\left(-\mathrm{CH}_{2}-\mathrm{CF}_{2}-\right)$ units have been replaced at random by $\mathrm{TrFE}\left(-\mathrm{CHF}_{-} \mathrm{CF}_{2}-\right)$ units. The arrows show the direction of the net dipole moments pointing from the fluorine side to the hydrogen side of the chain. b) Atomic-resolution STM image of a single-monolayer film of P(VDF-TrFE 70:30) deposited on graphite. ers on the basis of several observations: the new transition is strongest in the thinnest films; there is clear thermal hysteresis in the dielectric constant; and there are peaks in both the dielectric constant and the pyroelectric response. There is other evidence that the surface layers behave differently to the interior layers, because most films of $30 \mathrm{ML}$ or less are found to be partially or fully polarized immediately after fabrication, even before the application of an external electric field. Also, the polarization of the 2-7 ML films reverts in zero field to an orientation determined by the substrate, independent of the history of electric-field application $[8,9]$, consistent with the failure of the pyroelectric response to completely vanish above the Curie temperature in the thinnest films. These observations indicate that the surface layers have a preferred direction controlled by the interaction with the substrate or top electrode.
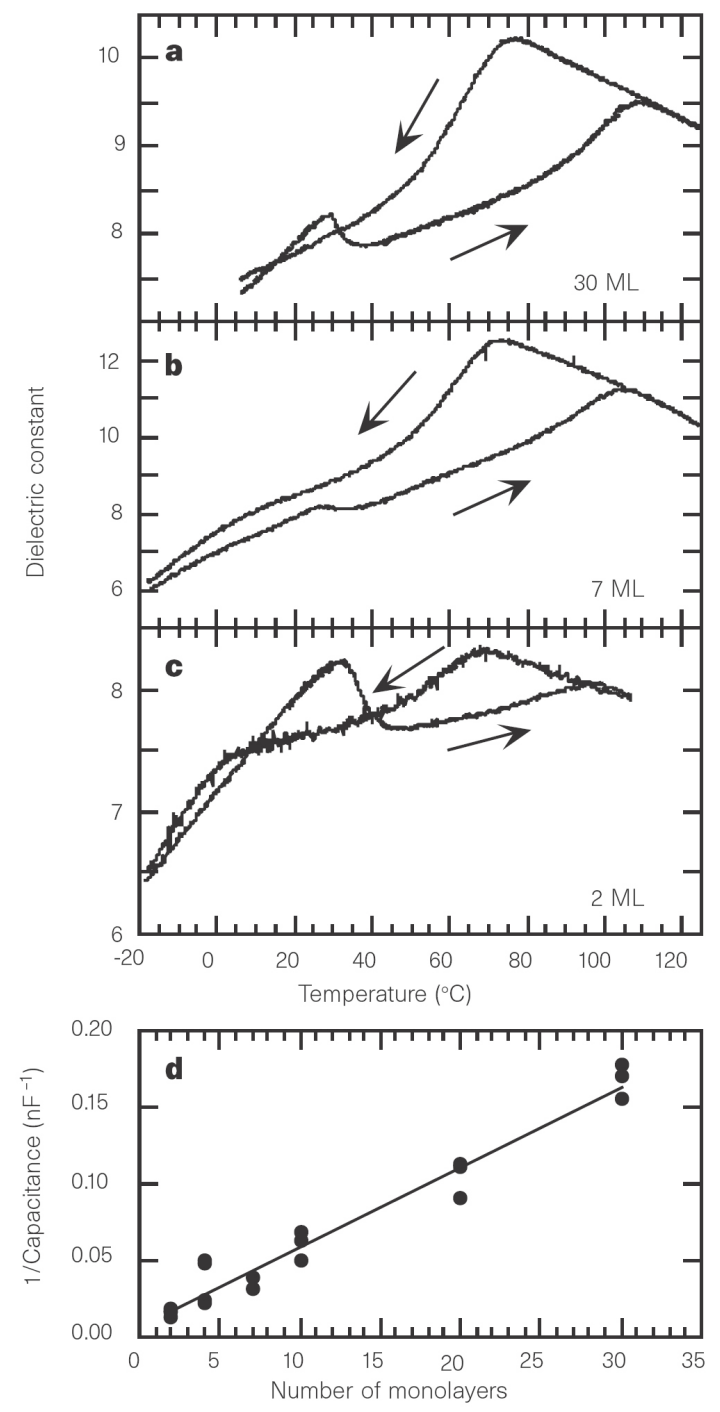

Figure 2. Dielectric properties of the FLP films. a-c, Dielectric constant $\varepsilon$ of the P(VDF-TrFE 70:30) films with different thicknesses, showing peaks at the phase transitions on heating and, due to thermal hysteresis, at different temperatures on cooling: a) $30 \mathrm{ML}$; b) $7 \mathrm{ML}$; c) $2 \mathrm{ML}$. The arrows show the direction of temperature change. d) The inverse capacitance at $25^{\circ} \mathrm{C}$ for FLP films with different thicknesses. 
The present results lead to the following conclusions. First, there is no apparent critical (minimum) thickness in FLP films as thin as $10 \AA$, much thinner than any previous ferroelectric films, with only a small suppression of the bulk Curie temperature. Second, there is a surface-layer ferroelectric phase transition at $\sim 20^{\circ} \mathrm{C}$ observable only in the thinnest FLP films. Therefore, the FLP films must be considered essentially twodimensional ferroelectrics because of the absence of strong finite-size effects; neither the coercive field nor the bulk phase transition temperature change significantly as the film thickness decreases from 150 to $10 \AA$, implying that the interlayer coupling is weak. Finite-size calculations based on a three-dimensional mean-field theory are not appropriate for modeling the thinnest FLP films with thickness comparable to size of the individual dipoles. The mean-field theory also predicts the existence of a critical thickness equal to the ferroelectric correlation length (a phenomenological parameter that is unknown in ferroelectric polymers [21]); the surface energy, connected with the truncation of the crystal and the dielectric properties of the external material, introduces another length scale. Similar considerations have been used to calculate [22] a critical size $\sim 25 \AA$ for small spherical particles of lead zirconate-titanate (PZT).

The two-dimensional nature of the FLP films means that the ferroelectric state may be generated by coupling only within the plane of the film. Any coupling between planes is weak and possibly responsible for the lower surface-layer $\mathrm{Cu}$ rie temperature. The Ising model for ultrathin films $[23,26]$ is a

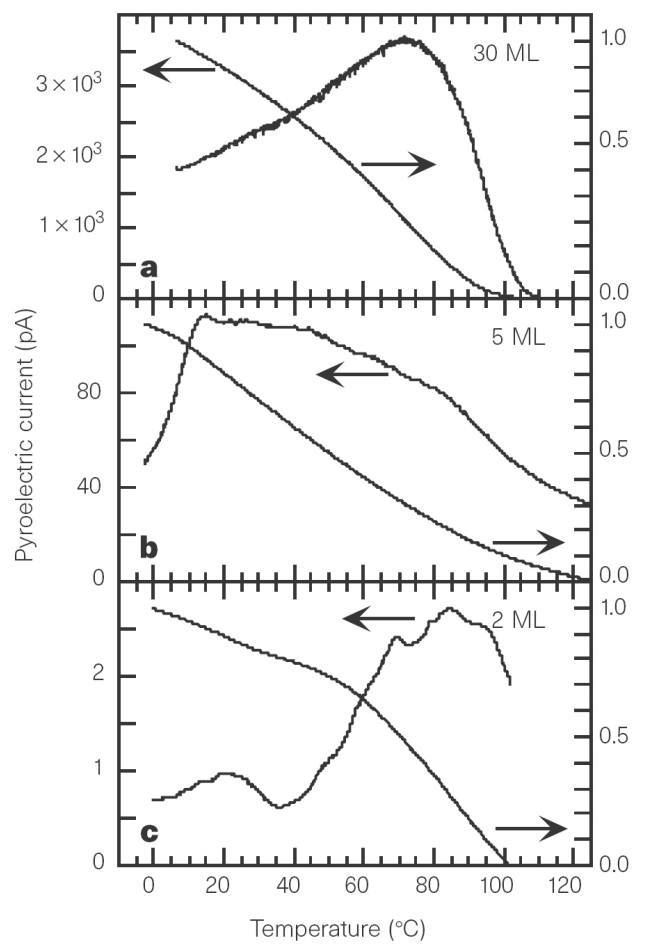

Figure 3. Pyroelectric response and spontaneous polarization $P_{s}$, obtained by integration over temperature, of P(VDF-TrFE 70:30) films. a) $30 \mathrm{ML}$; b) 5 $\mathrm{ML}$; c) $2 \mathrm{ML}$. more appealing approach to modeling ferroelectricity in twodimensional polymer films because the dipole moments have restricted freedom - they can rotate only about the chain axis and are further inhibited from rotations about the axis by both interchain steric interactions and intrachain dihedral stiffness. We expect that an appropriate Ising model could be constructed in either of two ways. First, use of anisotropic coupling constants (like the exchange integrals in ferromagnetism) with strong ferroelectric coupling in the plane and a weak coupling perpendicular to the plane. Second, use of a purely twodimensional model and making a weak inter-plane coupling through a mean-field shared by all layers. Both approaches can achieve ferroelectricity at finite temperature in a single layer and either enhancement or suppression by neighboring layers or electrodes, depending on the sign of the interlayer coupling. Both approaches also suggest that the surface layers, boundaries between the ferroelectric film and the electrodes or other outside material, have a Curie point different from the interior "bulk" layers because they couple with only one other ferroelectric layer. Fluctuations in two dimensions are not expected to destroy ordering as in the case of the isotropic Heisenberg ferromagnet [3] because the coupling is intrinsically anisotropic, and the polymer dipoles have close to one rotational degree of freedom, compared to the two rotational degrees of freedom of the magnetic dipoles.
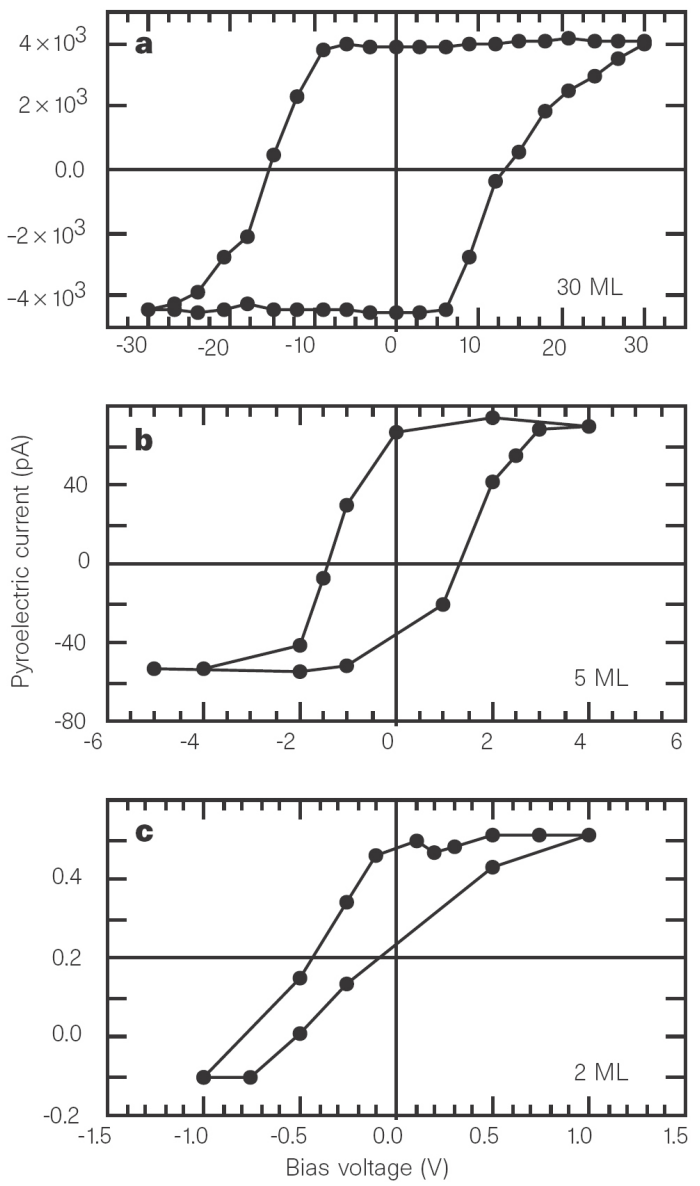

Figure 4. Polarization hysteresis loops at $25^{\circ} \mathrm{C}$, measured by the pyroelectric technique, of the $\mathrm{P}(\mathrm{VDF}-\mathrm{TrFE}$ 70:30) films with different thicknesses. a) $30 \mathrm{ML}$; b) $5 \mathrm{ML}$; c) $2 \mathrm{ML}$. 


\section{Methods}

The crystalline FLP films were formed by horizontal LangmuirBlodgett deposition from a water subphase, as reported in greater detail previously $[7,24]$. The STM image (Figure 1b) was obtained using an MTD (Moscow, Russia) instrument with a 1-ML FLP film deposited on a graphite substrate [25]. The films for electric measurements were deposited on aluminium-coated glass substrates and overcoated with aluminium evaporated in vacuum. The dielectric response (Figure 3) was measured with a commercial RLC meter (Hewlett-Packard 4192A) at $1 \mathrm{kHz}$ frequency and $0.1 \mathrm{~V}$ amplitude. The pyroelectric response at zero bias (Figure 2) and the hysteresis loops were measured by periodically heating the sample, with an $80-\mathrm{mW}$ laser beam operating at 514.5-nm wavelength and modulated at $2 \mathrm{kHz}$, and measuring the short-circuit current with a lock-in amplifier. The relative spontaneous polarization was obtained by integrating the pyroelectric current over temperature.

\section{Acknowledgments}

We thank P. A. Dowben and J. F. Scott for suggestions for improving the manuscript. Work at the University of Nebraska-Lincoln was supported by the USA NSF Division of Electronic and Communications Systems and by the Nebraska Research Initiative through the Center for Materials Research and Analysis. Work at the Institute of Crystallography was supported by INTAS.

\section{References}

I. Dürr,W., et al. Magnetic phase transition in two-dimensional ultrathin Fe films on Au(100). Phys. Rev. Lett. 62, 206-209 (1989).

2. Farle, M., and Baberschke, K. Ferromagnetic order and the critical exponent gamma for a Gd monolayer: an electron-spin-resonance study. Phys. Rev. Lett. 58, 5 I I-5 I4 (1987).

3. Mermin, N. D., and Wagner, H. Absence of ferromagnetism or antiferromagnetism in one- or two-dimensional isotropic Heisenberg models. Phys. Rev. Lett. 17, I I33-I I 36 (1966).

4. Dowben, P. A., Mcllroy, D. N., and Li, D. in Handbook on the Physics and Chemistry of Rare Earths (eds., Gschneidner, K. A., Jr., and Eyring, L.) Ch. 159 (Elsevier,Amsterdam, 1997).

5. Ishikawa, K., Yoshikawa, K., and Okada, N. Size effect on the ferroelectric phase transition in $\mathrm{PbTiO}_{3}$ ultrafine particles. Phys. Rev. B 37, 5852-5855 (1988).

6. Karasawa, J., Sugiura, M., Wada, M., Hafid, M., and Fukami, T. Ultra-thin lead titanate films prepared by tripole magnetron sputtering. Integrat. Ferroelectr. Lett. I 2, I05-I I 4 (1996).
7. Palto, S., et al. Ferroelectric Langmuir-Blodgett films. Ferroelectr. Lett. 19, 65-68 (1995).

8. Bune, A., et al. Novel switching phenomena in ferroelectric LangmuirBlodgett films. Appl. Phys. Lett. 67, 3975-3977 (1995).

9. Blinov, L. M., Fridkin, V. M., Palto, S. P., Sorokin, A.V., and Yudin, S. G.Thickness dependence of switching for ferroelectric Langmuir films. Thin Solid Films 284-285, 474-476 (1996).

10. Sorokin, A., Palto, S., Blinov, L., Fridkin, V., and Yudin, S. Ultrathin ferroelectric Langmuir-Blodgett films. Mol. Mater. 6, 6I-67 (1996).

I I. Scott, J. F. Phase transitions in ferroelectric thin films. Phase Trans. 30, I07-II0 (I99I).

12. Tilley, D. R. in Ferroelectric Thin Films: Synthesis and Basic Properties (eds., Paz de Araujo, C., Scott, J. F., and Taylor, G. F.) II-45 (Gordon \& Breach, New York, 1996).

13. Scott, J. F. Properties of ceramic $\mathrm{KNO}_{3}$ thin film memories. Physica $B$ I50, 160-167 (1988).

|4. Lovinger, A. J. Ferroelectric polymers. Science 220, I I I5-I I 2 I (I983).

I5.Wang, T.T., Herbert, J. M., and Glass, A. M. (eds.) The Applications of Ferroelectric Polymers (Chapman \& Hall, New York, 1988).

16. Furukawa, T. Ferroelectric properties of vinylidene fluoride copolymers. Phase Transit. 18, I43-2 I I ( I989).

17. Legrand, J. F. Structure and ferroelectric properties of P(VDF-TrFE) copolymers. Ferroelectrics 91, 303-317 (1989).

18. Kimura, K., and Ohigashi, H. Polarization behavior in vinylidene fluoride-trifluroethylene copolymer thin films. Jpn J. Appl. Phys. 25, 383387 (1986).

19. Ducharme, S. et al. Critical point in ferroelectric Langmuir-Blodgett polymer films. Phys. Rev. B 57, 25-28 (1998).

20. Allenspach, R., and Bishof, A. Magnetization direction switching in $\mathrm{Fe} /$ $\mathrm{Cu}(\mathrm{I} I 0)$ epitaxial films: temperature and thickness dependence. Phys. Rev. Lett. 69, 3385-3388 (1992).

2I. Qu, B. D., Zhang, P. L., Wang,Y. G.,Wang, C. L., and Zhong, W. L. Dielectric susceptibility of ferroelectric thin films. Ferroelectrics I52, 219224 (1994).

22. Yamamoto, T. Calculated size dependence of ferroelectric properties in $\mathrm{PbZrO}_{3}-\mathrm{PbTiO}_{3}$ system. Integr. Ferroelectr. 12, 16I-166 (1996).

23.Wang, C. L., Zhong,W. L., and Zhang, P. L.The Curie temperature of ultra-thin ferroelectric films.J. Phys.: Cond. Matter 3, $4743-4749$ (1992).

24. Blinov, L. M. Langmuir films. Sov. Phys. Usp. 3 I, 623-644 (1988).

25. Palto, S.et al. Ferroelectric Langmuir-Blodgett films showing bistable switching. Europhys. Lett. 34, 465-469 (1996).

26. Zhang, R., and Taylor, P. L. Theory of ferroelectric-paraelectric transitions in $\mathrm{VF}_{2} / \mathrm{F}_{4} \mathrm{E}$ random copolymers. J. Appl. Phys. 73, I395-I402 (1993). 